ARTICLE

\title{
Structure-based engineering of substrate specificity for pinoresinol-lariciresinol reductases
}

\author{
Ying Xiao ${ }^{1,8}$, Kai Shao 2,3,8, Jingwen Zhou (1) 4,5,8, Lian Wang ${ }^{4}$, Xueqi Ma1, Di Wu ${ }^{4}$, Yingbo Yang ${ }^{1}$, Junfeng Chen',

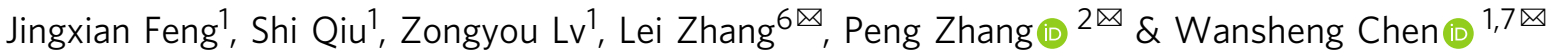

Pinoresinol-lariciresinol reductases (PLRs) are enzymes involved in the lignan biosynthesis after the initial dimerization of two monolignols, and this represents the entry point for the synthesis of 8-8' lignans and contributes greatly to their structural diversity. Of particular interest has been the determination of how differing substrate specificities are achieved with these enzymes. Here, we present crystal structures of liPLR1 from Isatis indigotica and pinoresinol reductases (PrRs) AtPrR1 and AtPrR2 from Arabidopsis thaliana, in the apo, substrate-bound and product-bound states. Each structure contains a head-to-tail homodimer, and the catalytic pocket comprises structural elements from both monomers. $\beta 4$ loop covers the top of the pocket, and residue 98 from the loop governs catalytic specificity. The substrate specificities of liPLR1 and AtPrR2 can be switched via structure-guided mutagenesis. Our study provides insight into the molecular mechanism underlying the substrate specificity of PLRs/PrRs and suggests an efficient strategy for the large-scale commercial production of the pharmaceutically valuable compound lariciresinol.

\footnotetext{
${ }^{1}$ Research and Development Center of Chinese Medicine Resources and Biotechnology, Institute of Chinese Materia Medica, Shanghai University of Traditional Chinese Medicine, Shanghai, China. ${ }^{2}$ National Key Laboratory of Plant Molecular Genetics, CAS Center for Excellence in Molecular Plant Sciences, Institute of Plant Physiology and Ecology, Chinese Academy of Sciences, Shanghai, China. ${ }^{3}$ University of Chinese Academy of Sciences, Beijing, China. ${ }^{4}$ National Engineering Laboratory for Cereal Fermentation Technology and School of Biotechnology, Jiangnan University, Wuxi, Jiangsu, China. ${ }^{5}$ Science Center for Future Foods, Jiangnan University, Wuxi, Jiangsu, China. ${ }^{6}$ Department of Pharmaceutical Botany, School of Pharmacy, Naval Medical University (Second Military Medical University), Shanghai, China. ${ }^{7}$ Department of Pharmacy, Changzheng Hospital, Naval Medical University (Second Military Medical University), Shanghai, China. ${ }^{8}$ These authors contributed equally: Ying Xiao, Kai Shao, Jingwen Zhou. ${ }^{凶}$ email: zhanglei@smmu.edu.cn; pengzhang01@cemps.ac.cn; chenwansheng@shutcm.edu.cn
} 
ignans are a major group of secondary metabolites in plants $^{1}$. This family has numerous biological effects in humans (e.g., anticancer ${ }^{2}$, antiviral ${ }^{3}$, antioxidant ${ }^{4}$, and immunosuppression ${ }^{5}$ ) owing to their structural diversity-nearly 2000 distinct lignans have been reported. For example, the furofuran lignans such as kandelisesquilignan A/B and terminaloside $\mathrm{K}$ have antioxidant effects ${ }^{6,7}$. The dibenzylbutyrolactone lignans including arctigenin, traxillagenin, arctiin, traxillaside, and their glycosides have neuroprotective activities ${ }^{8}$. Finally, the aryltetralin lignan podophyllotoxin ${ }^{9}$ is the precursor for the semi-synthesis of anticancer drugs such as etoposide ${ }^{10}$.

Lignans biosynthesis starts with the coupling of two coniferyl alcohols by an oxidase (laccase or peroxidase) with the aid of a dirigent protein to form pinoresinol ${ }^{11}$. Pinoresinol/lariciresinol reductase (PLR), an NADPH-dependent reductase, converts pinoresinol to lariciresinol and subsequently to secoisolariciresinol ${ }^{12}$. Because the reductive steps that give rise to lariciresinol and secoisolariciresinol represent entry points for the biosynthesis of the lignan subclasses furofurans, dibenzylbutane, dibenzylbutyrolactone, and aryltetrahydronaphthalene ${ }^{13}$, PLR is regarded as a pivotal enzyme that contributes to lignan structural diversity. Moreover, variation in both the composition and accumulation of lignans among different plant species, organs, and developmental stages can be ascribed, at least in part, to the characteristics of reactions catalyzed by PLRs as well as their expression patterns ${ }^{14}$. Therefore, characterization of the catalytic mechanisms of PLRs-especially their substrate selectivityis particularly crucial for understanding the molecular basis of the remarkable diversity of both chemical structures and biological activities of lignans.

The substrate selectivity of PLRs has attracted considerable attention $^{12}$. Most PLRs that have been characterized reduce both pinoresinol and lariciresinol efficiently to produce lariciresinol and secoisolariciresinol, respectively ${ }^{12}$. Known exceptions are Arabidopsis thaliana reductases that have substrate preference for pinoresinol, but only weak (AtPrR1) or no activity (AtPrR2) toward lariciresinol and are thus named pinoresinol reductases (PrRs) ${ }^{15}$. A recent study indicated that the L174I mutant of Camellia sinensis PLR1 (CsPLR1) loses the capacity to reduce pinoresinol and specifically catalyzes the conversion of lariciresinol to secoisolariciresinol, but the underlying mechanism is unclear $^{16}$. The three-dimensional structure of Thuja plicata PLR1 (TpPLR1) has been elucidated and indicates that $\mathrm{K} 138$ is responsible for the basic catalysis, because the mutant K138A lacks the ability to convert pinoresinol ${ }^{17}$. However, the apo structure does not provide sufficient information to interpret the substrate-selective mechanism of PLRs/PrRs.

Phylogenetic analysis of PLRs/PrRs from different species has revealed that Isatis indigotica PLR1 (IiPLR1), a key enzyme involved in lariciresinol biosynthesis, has the closest relationship to AtPrRs with a high level of amino-acid sequence identity $(>80 \%)^{18}$ and is grouped with AtPrR2, which cannot utilize lariciresinol as substrate (Fig. 1). Interestingly, in contrast to AtPrRs that has substrate preference for pinoresinol, IiPLR1 from $I$. indigotica (family Cruciferae, same as A. thaliana) can reduce both pinoresinol and lariciresinol efficiently with comparable $k_{\text {cat }} /$ $K_{\mathrm{m}}$ values $^{18}$. The finding that IiPLR1/AtPrRs, which differ in substrate selectivity, are clustered together suggests that substrate specificity is independent of sequence conservation among PLRs/ PrRs. Therefore, the amino-acid residues responsible for PLR/PrR substrate selectivity are difficult to determine merely through sequence analysis, and thus structural information on PLR/PrR enzymes is vital-as are data concerning how these two enzyme types can utilize two different substrates.

In the present work, we compare crystal structures of IiPLR1, AtPrR1, and AtPrR2 and identify residues that may be responsible for the observed substrate selectivity of PLRs and PrRs.
Mutagenesis of these residues alters the substrate specificities for pinoresinol and lariciresinol. For example, mutagenesis of IiPLR1 successfully eliminates the second reaction that converts lariciresinol to secoisolariciresinol, leading to a high accumulation of the pharmaceutically valuable compound lariciresinol. Our study will enable the synthesis of lignans with diverse chemical structures and bioactivities by biotechnological means or by enzyme-assisted chemistry.

\section{Results}

Characterization of IiPLR1, AtPrR1, and AtPrR2 crystal structures. To understand both the catalytic mechanism of PLR and the mechanism underlying the substrate specificity of PLRs/ PrRs, we chose IiPLR1, AtPrR1, and AtPrR2 for a structure study. The crystal structures were captured in the apo, substrate-bound and/or product-bound forms (Table 1). We found that, for all 16 structures we solved, each enzyme adopts a similar head-to-tail dimer conformation (Fig. 2a and Supplementary Fig. 1), strongly suggesting that each PLR/PrR functions as a homodimer, consistent with the literature that TpPLR1 exists as a dimeric entity in solution $^{17}$

Taking the structure of IiPLR1_NAP_+PIN for the purpose of a detailed description, each protomer contains two domains, namely the N-terminal NADPH binding domain (NBD) and the C-terminal substrate binding domain (SBD). The NBD comprises seven $\beta$-strands $(\beta 1-6, \beta 8)$ surrounded by six $\alpha$-helices $(\alpha 1-5$, $\alpha 7)$, whereas the SBD comprises two $\beta$-strands $(\beta 7, \beta 9)$ with five small $\alpha$-helices $(\alpha 6, \alpha 8-11)$. A large groove is formed between NBD and SBD (Fig. 2a). This groove can be roughly divided into two parts-the positively charged part that associates with the NBD and the hydrophobic part that associates with the SBD. The substrates or products can be clearly defined within the groove (Fig. 2a and Supplementary Fig. 2). Several regions ( $\alpha 5$ loop, $\alpha 9$ helix, and a9 loop) are partially disordered both in the IiPLR1_apo and IiPLR1_NAP structures, for which the differences can be characterized by a RMSD of $0.287 \AA$, whereas the $\beta 4$ loop is well defined in the apo structure (Fig. 2b). Intriguingly, the $\beta 4$ loop is well defined in the AtPrR1_NAP structure but disordered in the AtPrR1/2_apo structures (Supplementary Fig. 3). These structural differences suggest that the loops are somewhat flexible, and act as a switch to control the binding of $\mathrm{NADPH}$ and release of $\mathrm{NADP}^{+}$. Moreover, the $\beta 2$ loop moves slightly towards $\mathrm{NADP}^{+}$in the IiPLR1_NAP structure, catering to the entry of the coenzyme (Fig. $2 \mathrm{~b}$ ). $\mathrm{NADP}^{+}$forms strong hydrogen bonds and hydrophobic interactions with residues within the groove (Fig. 2c), among which the GXXGXXG motif (considered as the conserved NADPH-binding motif) binds the phosphate and deoxyribose groups, and residues Ala164, Cys165 together with Phe166 fix the position of the catalytically active nicotinamide group. Residue Lys144, which corresponds to the previously reported Lys138 in TpPLR1 that serves as the general base for catalysis, forms direct hydrogen bonds with $\mathrm{NADP}^{+}$in IiPLR1_apo and IiPLR1_NAP.

Catalytic mechanism of PLR based on its homodimeration. The dimers of IiPLR1_NAP and IiPLR1_NAP_+ PIN have similar structures, as suggested by a RMSD of $0.362 \AA$, indeed, even the $\mathrm{NADP}^{+}$moieties could be aligned almost in the same position (Fig. 2d). Both $\beta 4$ loops are disordered, further implying their flexibility, but $\beta 2$ loop and $\alpha 10$-helix from neighboring molecules of IiPLR1_NAP_ + PIN dimer make contacts with and stabilize the substrate (Fig. 2d, e). Similar conformational changes of $\beta 2$ loop and a10-helix can be seen by comparing the structure of AtPrR1_NAP_+ PIN with that of AtPrR1_NAP and the structure of AtPrR2_NAP_+PIN with that of AtPrR2_apo 
a<smiles>COc1cc(C2CC3C(CO2)COC3c2ccc(O)c(OC)c2)ccc1O</smiles>

Pinoresinol

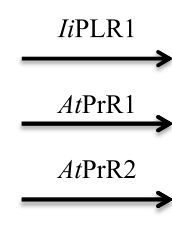

Lariciresinol

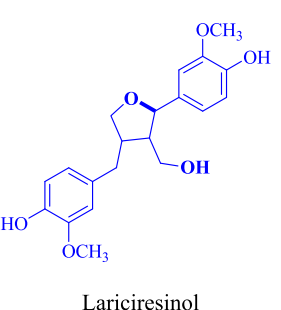

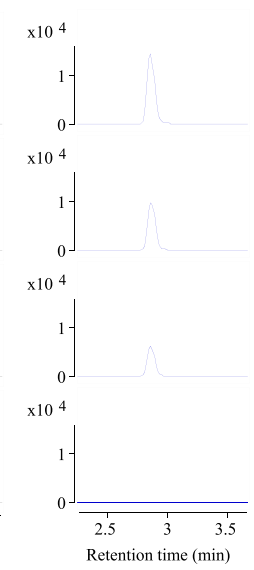
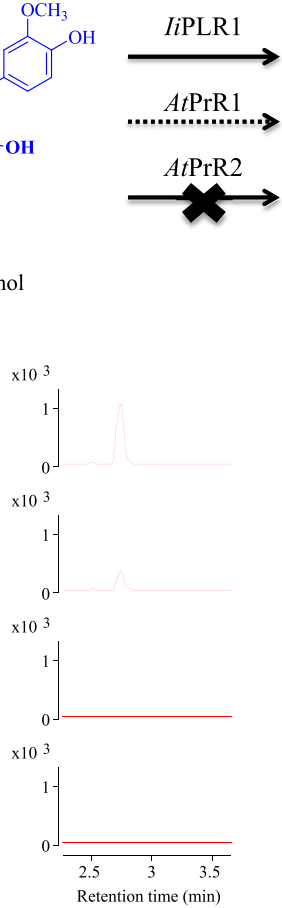

C

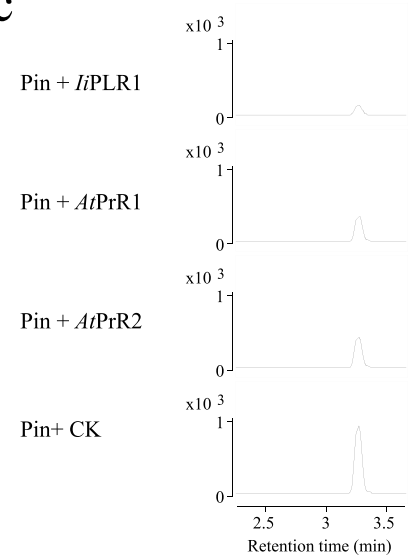

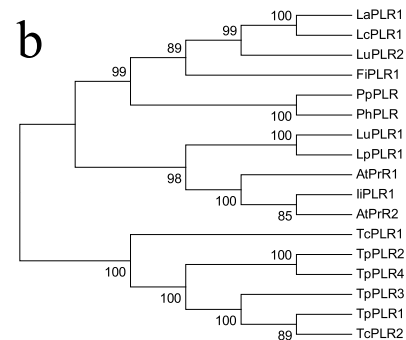

Secoisolariciresinol

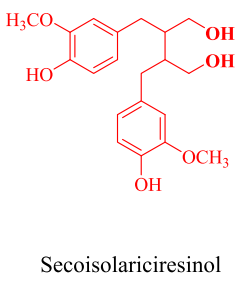

$\mathrm{d}$
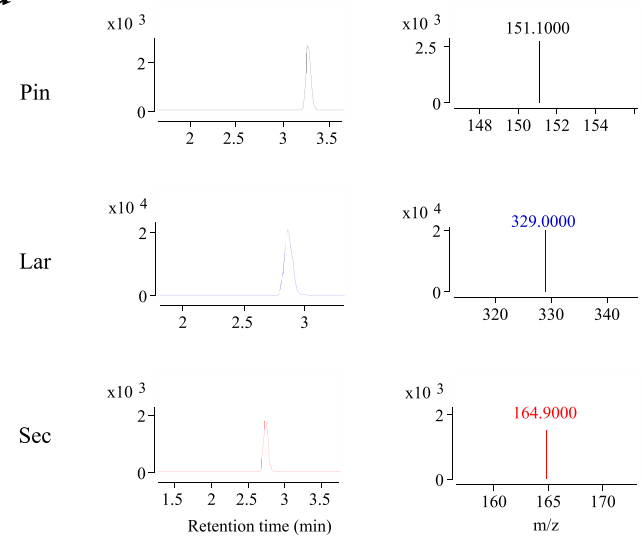

Fig. 1 Biochemical assays for liPLR1 and AtPrRs function. a liPLR1 efficiently catalyzes the conversion of pinoresinol into lariciresinol and also catalyzes the conversion of lariciresinol into secoisolariciresinol. In contrast, AtPrR1/2 exhibit a substrate preference for pinoresinol, yet exhibit only weak activity (AtPrR1) or no activity (AtPrR2) for lariciresinol. b Phylogenetic tree of PLRs/PrRs from different species. c Conversion of pinoresinol into lariciresinol and then into secoisolariciresinol by recombinant liPLR1, AtPrR1, and AtPrR2. The reaction products were analyzed by LC-MS. d Chromatograms for pinoresinol, lariciresinol, and secoisolariciresinol are denoted in black, blue, and red, respectively.

(Supplementary Fig. 4a, b). (+)-Pinoresinol is inserted as a straight chain deep into the hydrophobic groove, for which the hydrophilic ends are stabilized through the formation of hydrogen bonds with main-chain atoms of Met125 and Gly178, and the hydrophobic region is surrounded by a series of hydrophobic groups (Fig. 2e). The inner 2-methoxy-phenol group of $(+)$-pinoresinol forms a sandwich-like $\pi-\pi$ stack comprising the nicotinamide head of $\mathrm{NADP}^{+}$and Phe166. Two furan rings in the middle are surrounded by Tyr169 and Phe170 from a6-helix and by His276 and Phe277 from a10-helix. The outer 2-methoxyphenol group is coordinated by Phe277 and Val46 of $\beta 2$ loop from a neighboring protomer, which is distant from the $\mathrm{NADP}^{+}$ and (+)-pinoresinol of the protomer (Fig. 2a, c, e). Further, Lys144 is far away from the furan rings, indicating that it may not participate in catalysis directly. Based on this structure analysis, we propose that both the entry and exit of NADPH are controlled by the $\beta 4$ loop of IiPLR1. Once one molecule of $(+)$-pinoresinol is captured by the narrow hydrophobic groove, each protomer forces the prepositioning of the $\alpha 10$-helix and $\beta 2$ loop in the other protomer, resulting in tight binding of the substrate. This allows $\mathrm{H}$ : transfer from the NADPH to the proximal furan ring of the substrate to produce one molecule of (+)-lariciresinol.

Regarding the mechanism of the second catalytic step, we further compared the structures of IiPLR1_NAP_+ PIN, IiPLR1_NAP_-LAR and IiPLR1_NAP_-SEC, which revealed a similar mode for substrate/product binding (Fig. 2f). Furthermore, Leu46 (corresponding to Val46 in IiPLR1), His276 and Phe277 of AtPrR1 are positioned similar to the corresponding residues of IiPLR1 to effect substrate binding or product release, except that the $\beta 4$ loops cover the substrate/product, which are disordered in the IiPLR1 structures (Fig. 2d and Supplementary Fig. 4a, c, d). The importance of Val46 for catalysis in IiPLR1 is underscored by data from a mutational analysis (Fig. 2g).
Mutation of Val46 to Ala improved the conversion of pinoresinol to lariciresinol by $\sim 16 \%$, and the subsequent conversion to secoisolariciresinol was greatly reduced. The I $i$ PLR1 mutant V46L had little ability to catalyze the conversion of lariciresinol to secoisolariciresinol. These data suggest that IiPLR1 undergoes substrate-induced conformational changes upon homodimerization to achieve catalysis, and the principle of catalytic reactions using lariciresinol as substrate (second step) appears to be like that using pinoresinol as substrate (first step).

Mechanism underlying the substrate selectivity of PLR/PrR. A previous study reported that the recombinant AtPrR1 can only weakly reduce lariciresinol whereas AtPrR2 lacks activity, which is in sharp contrast to all known PLRs ${ }^{15}$. To determine the mechanism underlying this difference in substrate specificity, we confirmed the relative lack of activity for $A t \operatorname{PrR} 1 / 2$ (Fig. 1) and then carried out a structure analysis of IiPLR1, AtPrR1, and AtPrR2. Each of IiPLR1_NAP_+ PIN/AtPrR1_NAP_+ PIN/ AtPrR2_NAP_+ PIN forms a homodimer, and superimposition of the protomers among the three complexes revealed RMSDs of $0.374,0.365$, and $0.308 \AA$, respectively (Fig. 3a, c and Supplementary Fig. 1). In contrast to IiPLR1_NAP_+ PIN, the $\beta 4$ loops of AtPrR1_NAP_+ PIN and AtPrR2_NAP_ + PIN can be clearly identified (Fig. 3 and Supplementary Fig. 4 ). These well-defined loops twist as an " 8 " shape and cover both the $\mathrm{NADP}^{+}$-binding and substrate-binding grooves. Within the twisted loop, His93 and His97 "grasp" helices $\alpha 5$ and $\alpha 10$, while Val92 and Phe94 interact directly with (+)-pinoresinol; Arg95 strongly interacts with NADP ${ }^{+}$as well as each of the GXXGXXG motif and a2helix from neighboring protomer of the dimer. Similar $\beta 4$ loops are also observed in all other AtPrR1 substrate/product-bound structures, whereas each $\beta 4$ loop is disordered in the 


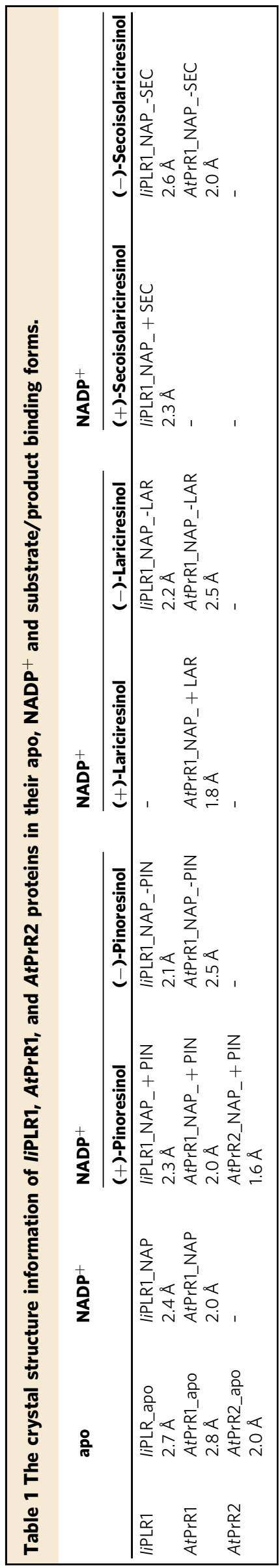

corresponding IiPLR1 structures, which indicates that the $\beta 4$ loop may participate in substrate selectivity and, hence, catalysis.

We further explored why the loops behaved differently between IiPLR1 and the AtPrRs. The amino acid sequences of the $\beta 4$ loops in the three proteins are quite similar (Supplementary Fig. 5), but the residue corresponding to Ser98 at the C-terminal end of the loop in IiPLR1 is replaced as Asn98 in AtPrR1/2. Combining the sequence and structural data, the difference can be explained reasonably as follows: the serine side chain is short enough to remain beneath the guanine group of $\mathrm{NADPH}$, whereas the asparagine side chain cannot do so owing to steric hindrance. Consequently, the asparagine lies nearly vertical to the guanine group and points upward in the structure shown in Fig. 3b, d, and thus the swing of the $\beta 4$ loop is limited in the AtPrR1_NAP structure. As substrate enters the catalytic site, $\beta 4$ loop can fold and cover the substrate-binding groove (Fig. $3 \mathrm{~b}, \mathrm{~d}$ and Supplementary Figs. $3 \mathrm{~b}$ and $4 \mathrm{a}$ ). Besides the $\beta 4$ loop, Val46 in IiPLR1 is replaced with Leu46 in AtPrR1, which has the effect of compressing the substrate-binding pocket. Although Val46 is unchanged in AtPrR2, the a2-helix and $\beta 2$ loop from the neighboring protomer move closer to the substrate upon its entry at the catalytic site, further condensing the pocket. The relative movement of dimers between AtPrR2 and IiPLR1 (as suggested by the $\sim 9^{\circ}$ shift shown in Fig. 3c) could be induced by different dimer orientations. Two molecules of the dimer exhibit relative torsion in AtPrR2, and consequently, Val46 is forced deeper into the substrate-binding pocket compared with what occurs in IiPLR1. Therefore, the entrance and orientation of the substrate in $A t \operatorname{PrR} 1 / 2$ is more tightly controlled than in IiPLR1.

Mutagenesis-based alteration of the substrate selectivity. Based on the structural analysis of IiPLR1, AtPrR1 and AtPrR2, the importance of the candidate amino acids controlling substrate specificity was verified through site-directed mutagenesis. Enzymatic assays using pinoresinol as substrate revealed that the IiPLR1 mutations including V46A, V46L, S98A, S98H, and S98N somewhat enhanced the conversion rate of lariciresinol while significantly reduced that of secoisolariciresinol, and mutants V46A, S98A, and S98H had $>40 \%$ conversion rates for lariciresinol (Fig. 4a), suggesting that residues 46 and 98 are critical for the substrate preference. Taking V46A as an example for the kinetic analysis, its $K_{\mathrm{m}}$ value for pinoresinol $(29.4 \pm 1.62 \mu \mathrm{M})$ is comparable with that for lariciresinol $(26.5 \pm 0.60 \mu \mathrm{M})$, however its $V_{\max }$ for pinoresinol $\left(3.22 \pm 0.68 \mu \mathrm{M} \mathrm{min}^{-1}\right)$ is 140 -fold higher than that for lariciresinol $\left(0.023 \pm 0.0013 \mu \mathrm{M} \mathrm{min}^{-1}\right)$, and its $k_{\mathrm{cat}} /$ $K_{\mathrm{m}}$ for pinoresinol $\left(3.88 \pm 0.65 \mu \mathrm{M}^{-1} \mathrm{~min}^{-1}\right)$ is 126 -fold higher than that for lariciresinol $\left(0.031 \pm 0.002 \mu \mathrm{M}^{-1} \mathrm{~min}^{-1}\right)$ (Table 2). Compared with wild-type IiPLR $1^{18}$, the activity of mutant V46A towards pinoresinol increases 4 -fold, whereas that towards lariciresinol decreases $98 \%$ with regard to $k_{\text {cat }} / K_{\mathrm{m}}$ values. These results indicate mutant V46A enhances catalytic efficiency for the first reaction but dramatically eliminates the second reaction. Consistent with the data for IiPLR1, AtPrR1 mutants L46A and L46V could enhance the conversion rate of lariciresinol and partially reduce that of secoisolariciresinol (Fig. 4a), which confirmed the importance of these two sites in substrate binding and product release thus in catalysis. As expected, mutants N98A and N98S in AtPrR1 had increased activity for secoisolariciresinol production compared with wild type (Fig. 4a), strongly implying that residue 98 controls the swing of the $\beta 4$ loop, which affects substrate binding and catalysis. Interestingly, AtPrR2 mutant N98S could utilize lariciresinol to produce secoisolariciresinol, with a conversion rate of $1.91 \%$, in contrast to the wild type which lacks this activity (Fig. 4a). Other AtPrR2 mutants, including V46A, V46L, and N98A, varied in their activities for pinoresinol, 
a

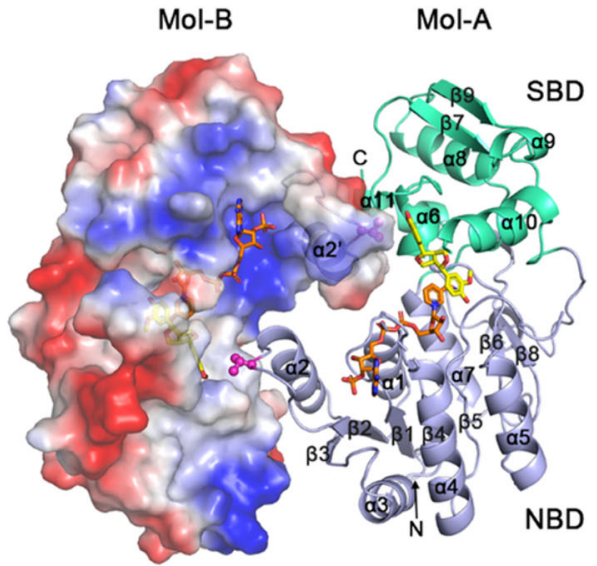

b

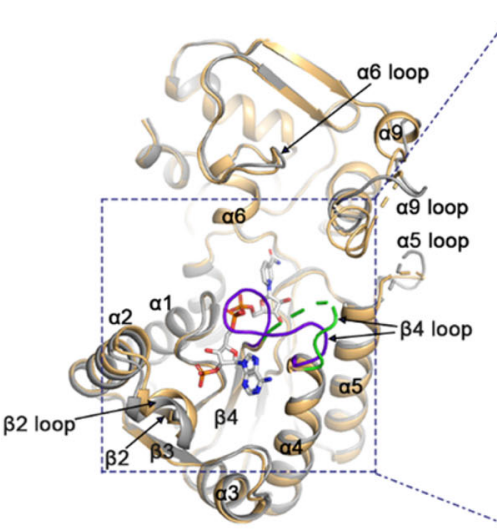

C

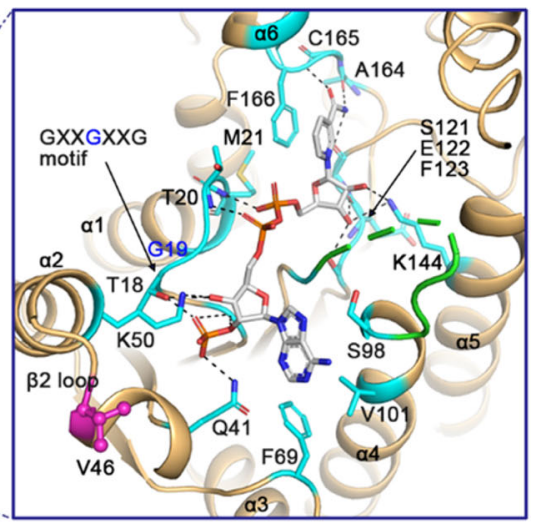

d

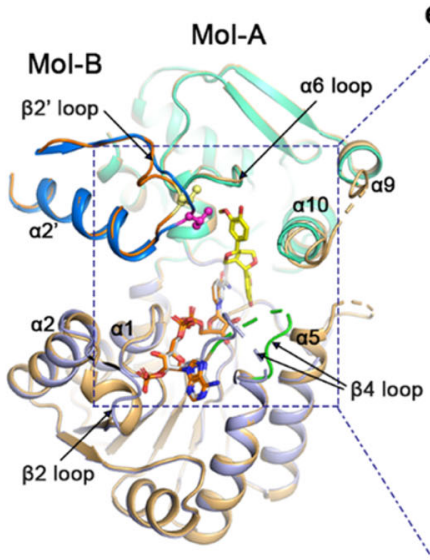

e

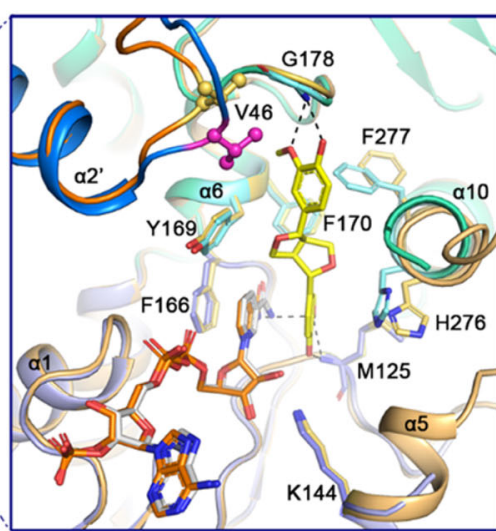

$f$

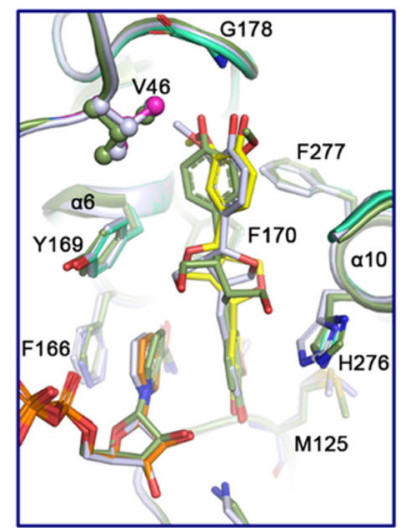

g

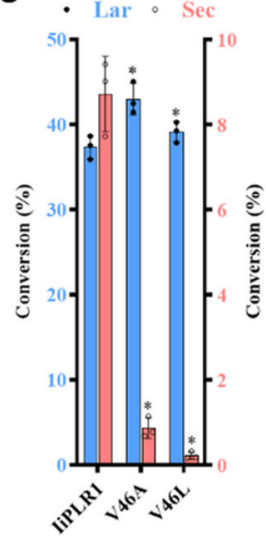

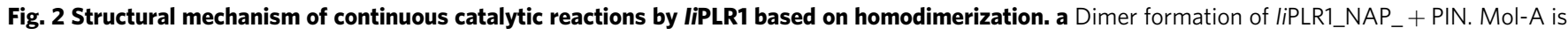
shown as cartoon model, with its NBD and SBD colored in light blue and green cyan, respectively. Mol-B is represented as an electrostatic-surface model, on which blue and red colors represent positive and negative charges, respectively. NADP + and ( + )-pinoresinol are shown as sticks and colored in orange and yellow, respectively. b Conformational changes were assessed by comparing monomer structures of liPLR1_apo (gray) and liPLR1_NAP (light orange). The NADP + bound to liPLR1_NAP is colored gray. The $\beta 4$ loops of liPLR1_apo and liPLR1_NAP are highlighted as purple and green, respectively. c Zoom-in view of the NADPH-binding groove of liPLR1_NAP. Residues interacting with NADP+ are colored cyan. The conserved GXXGXXG motif is indicated. Residue Val46 involved in dimer formation and substrate binding is shown as a ball-and-stick model and colored magenta. Dotted lines denote possible hydrogen bonds. d Structure comparison of liPLR1_NAP_+ PIN and liPLR1_NAP. Mol-Bs of liPLR1_NAP_+ PIN and liPLR1_NAP, Val46s in liPLR1_NAP_+

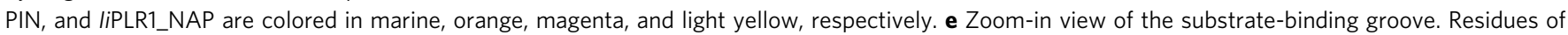
Mol-As in liPLR1_NAP_+ PIN and liPLR1_NAP are colored green cyan (or light blue) and light yellow, respectively. $\mathbf{f}$ Structural comparation of the substrate/product-binding grooves in liPLR1_NAP_+ PIN, liPLR1_NAP_-LAR (blue white), and liPLR1_NAP_-SEC (smudge). The cartoons are generated by PyMOL. $\mathbf{g}$ Enzyme assays for wild-type liPLR1 and its mutants V46A and V46L. Data are mean \pm s.d. ( $n=3$ independent experiments). Asterisk * indicates

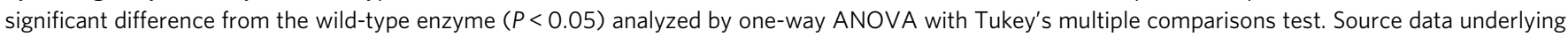
Fig. $2 \mathrm{~g}$ are provided as a Source Data file.

as indicated by the relative rates of conversion to lariciresinol (Fig. 4a). Similar results were obtained for conversion of lariciresinol to secoisolariciresinol (Fig. 4b). Altogether, the structureguided mutagenesis indeed could switch the substrate specificity of PLR/PrR, e.g., the IiPLR1 mutant V46A had increased preference for pinoresinol but little catalytic activity for lariciresinol, whereas the AtPrR2 mutant N98S gained the activity to catalyze the conversion of lariciresinol to secoisolariciresinol (Fig. 4c).

Taking structure and enzymology data together, we proposed a three-step catalytic mechanism for PLR based on its homodimerization. First, the protomers of dimeric PLRs recruit free NADPH through the very flexible $\beta 4$ loop. Second, pinoresinol binds into one protomer via the substrate-binding groove, and the other protomer of the homodimer helps stabilize the substrate. Subsequently, pinoresinol receives $\mathrm{H}$ : from $\mathrm{NADPH}$ and be reduced to lariciresinol released later. Third, free lariciresinol is bound by another reductive PLR molecule and fixed by another homodimer, and then the lariciresinol is reduced to secoisolariciresinol and finally released (Fig. 5 and Supplementary Movie 1).

Importantly, the PrRs have more strict requirements for the binding and orientation of lariciresinol compared with PLRs, so PrRs cannot efficiently carry out the third step (Fig. 5). Hence, the substrate-specificity mechanism of PLRs/PrRs could be that residues located around the substrate-binding pocket and within the loop, together with residues that promote homodimerization, form the appropriate hydrophobic environment for binding a specific substrate.

Mutation increases lariciresinol and reduces secoisolariciresinol production in vivo. Enzymatic assays indicated that certain IiPLR1 mutants had increased activity for producing lariciresinol from pinoresinol in vitro (Fig. 4). Therefore, these IiPLR1 mutant genes were selected for lariciresinol production using pinoresinol-producing E. coli ${ }^{19}$. Because matairesinol, 

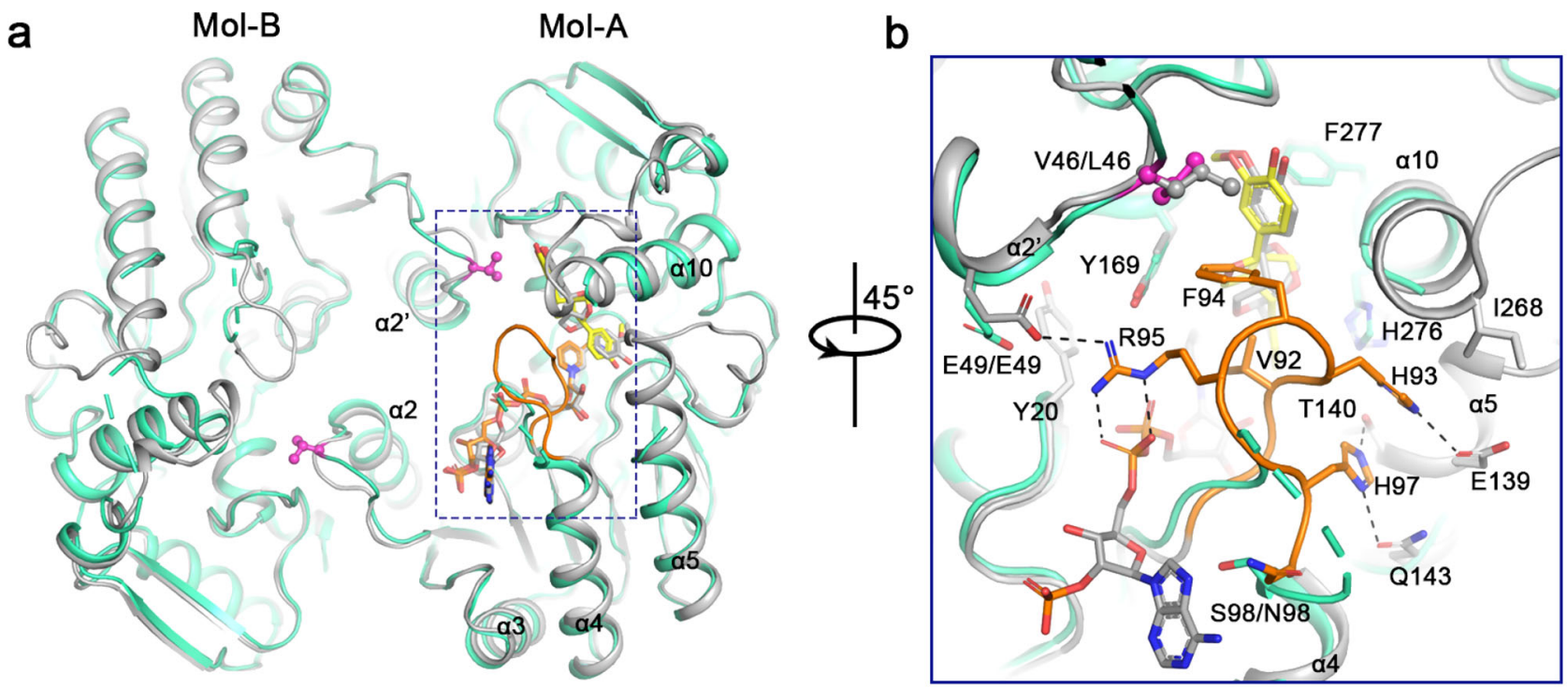

C
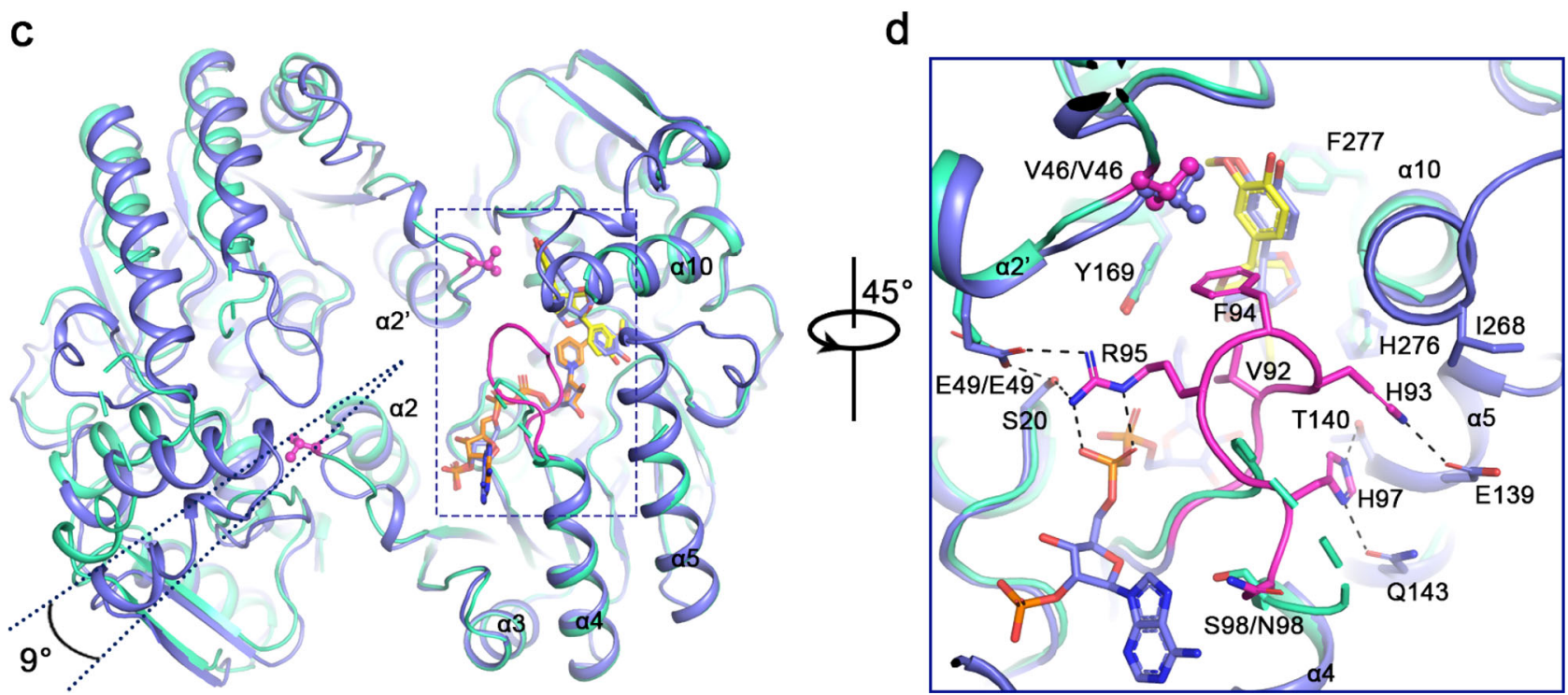

Fig. 3 Structural differences among liPLR1, AtPrR1, and AtPrR2 indicating different catalytic capacities. a Structural alignment of dimerized liPLR1_NAP_+ PIN (green cyan) and AtPrR1_NAP_+ PIN (light gray). b Zoom-in view of dashed box in a. The $\beta 4$ loop of AtPrR1_NAP_+ PIN is highlighted in orange. c Structural alignment of dimerized liPLR1_NAP_ + PIN (green cyan) and AtPrR2_NAP_+ PIN (slate blue). d Zoom-in view of the dashed box in c. The $\beta 4$ loop of AtPrR2_NAP_+ PIN is highlighted in magenta. For brevity, NADP+ and some residues in liPLR1 are hidden. The cartoons are generated by PyMOL.

which is derived from secoisolariciresinol, is detectable only when CueO (multicopper oxidase), PLR and SDH (secoisolariciresinol dehydrogenase) are individually expressed in cells ${ }^{20}$, each of wildtype IiPLR1 and its mutants were co-cultured in pinoresinolproducing $E$. $\operatorname{coli}^{19}$. Consistent with the enzyme assay results, IiPLR1_V46A produced the greatest amount of lariciresinol (997.79 $\mathrm{mg} \mathrm{L}^{-1}$ compared with $936.14 \mathrm{mg} \mathrm{L}^{-1}$ for wild-type). However, mutants V46L, S98A, S98H, and S98N were not as efficient as wild-type cells at producing lariciresinol, which was opposite to the results from in vitro enzyme assays. This may reflect the potential effects of complex metabolic networks and feedback mechanisms in vivo, which are not relevant to in vitro enzyme assays. Moreover, the provision of NADPH is tightly regulated in prokaryotic systems, which also may influence the activity of PLRs.

Notably, all the IiPLR mutants produced significantly less secoisolariciresinol than wild-type cells, i.e., by $22.7-52.5 \%$; in particular, IiPLR_V46A produced $46.4 \%$ less secoisolariciresinol than wild type (Fig. 6). These results paralleled those obtained in vitro with the IiPLR1 mutants in which there was elimination of the second catalytic step, i.e., the conversion of lariciresinol to secoisolariciresinol (Fig. 4). Taken together, our results establish a promising route for the production of lariciresinol by synthetic biology strategies, and mutant IiPLR_V46A mutant would be a good candidate for use in the large-scale production of the pharmaceutically valuable compound lariciresinol.

\section{Discussion}

The molecular mechanism of substrate selectivity of PLR/PrR has attracted particular interest owing to the key role of these enzymes in lignan biosynthesis. However, the lack of structural results-especially for PLRs/PrRs in complex with different substrates-has limited our understanding of the mechanism underlying enzyme specificity. In the present study, we characterized crystal structures of IiPLR1, AtPrR1, and AtPrR2 in 
$\mathbf{a}$

Pin as Substrates

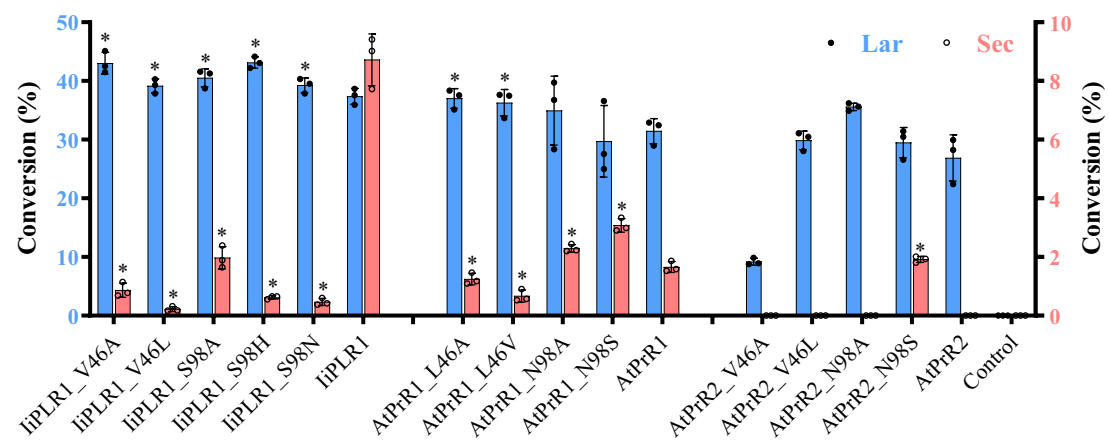

b

Lar as Substrate
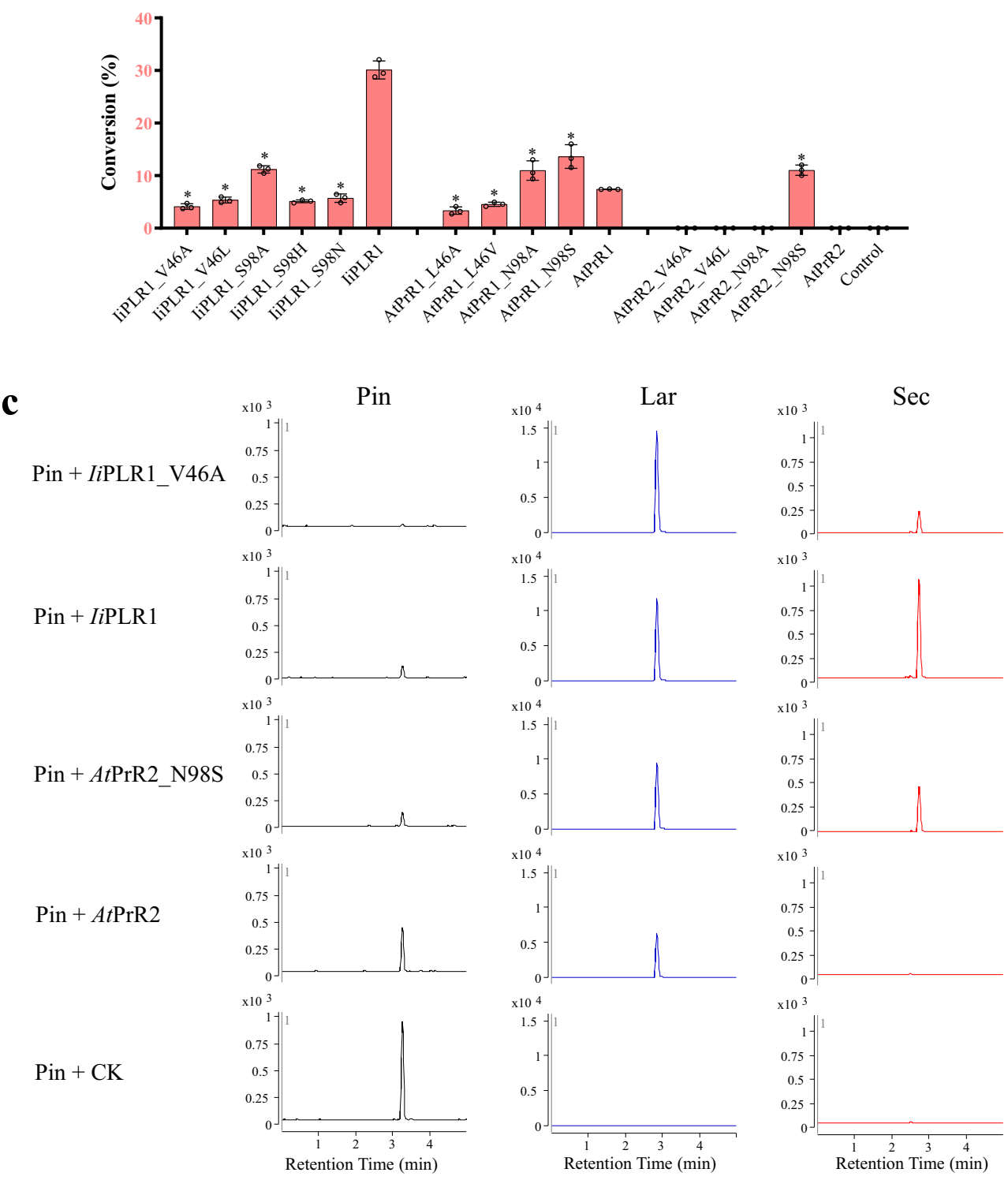

Fig. 4 Percent conversion of pinoresinol to lariciresinol and subsequently to secoisolariciresinol by mutants of liPLR1, AtPrR1 and AtPrR2. a

Conversion of pinoresinol into lariciresinol and subsequently to secoisolariciresinol. $\mathbf{b}$ Conversion of lariciresinol into secoisolariciresinol. Data are mean \pm s.d. ( $n=3$ independent experiments). Asterisk $\left(^{*}\right)$ indicates significant difference from the wild-type enzyme $(P<0.05)$ analyzed by one-way ANOVA with Tukey's multiple comparisons test. Source data underlying Fig. $4 a, b$ are provided as a Source Data file. c LC-MS determination of the products as catalyzed by liPLR1, liPLR1_V46A, AtPrR2 and AtPrR2_N98S. 
Table 2 Kinetic properties of liPLR1_V46A.

\begin{tabular}{|c|c|c|c|c|}
\hline Substrate & $K_{m}(\mu M)$ & $V_{\max }(\mu \mathrm{M} / \mathrm{min})$ & $k_{\text {cat }}\left(\min ^{-1}\right)$ & $k_{\mathrm{cat}} / K_{\mathrm{m}}\left(\mu \mathbf{M}^{-1} \min ^{-1}\right)$ \\
\hline ( \pm )-pinoresinol & $29.4 \pm 1.62$ & $3.22 \pm 0.68$ & $114.7 \pm 24.1$ & $3.88 \pm 0.65$ \\
\hline$( \pm)$-lariciresinol & $26.5 \pm 0.60$ & $0.023 \pm 0.0013$ & $0.81 \pm 0.045$ & $0.031 \pm 0.002$ \\
\hline
\end{tabular}

Data are expressed as mean \pm s.d. with three independent experiments. Source data are provided as a Source Data file.

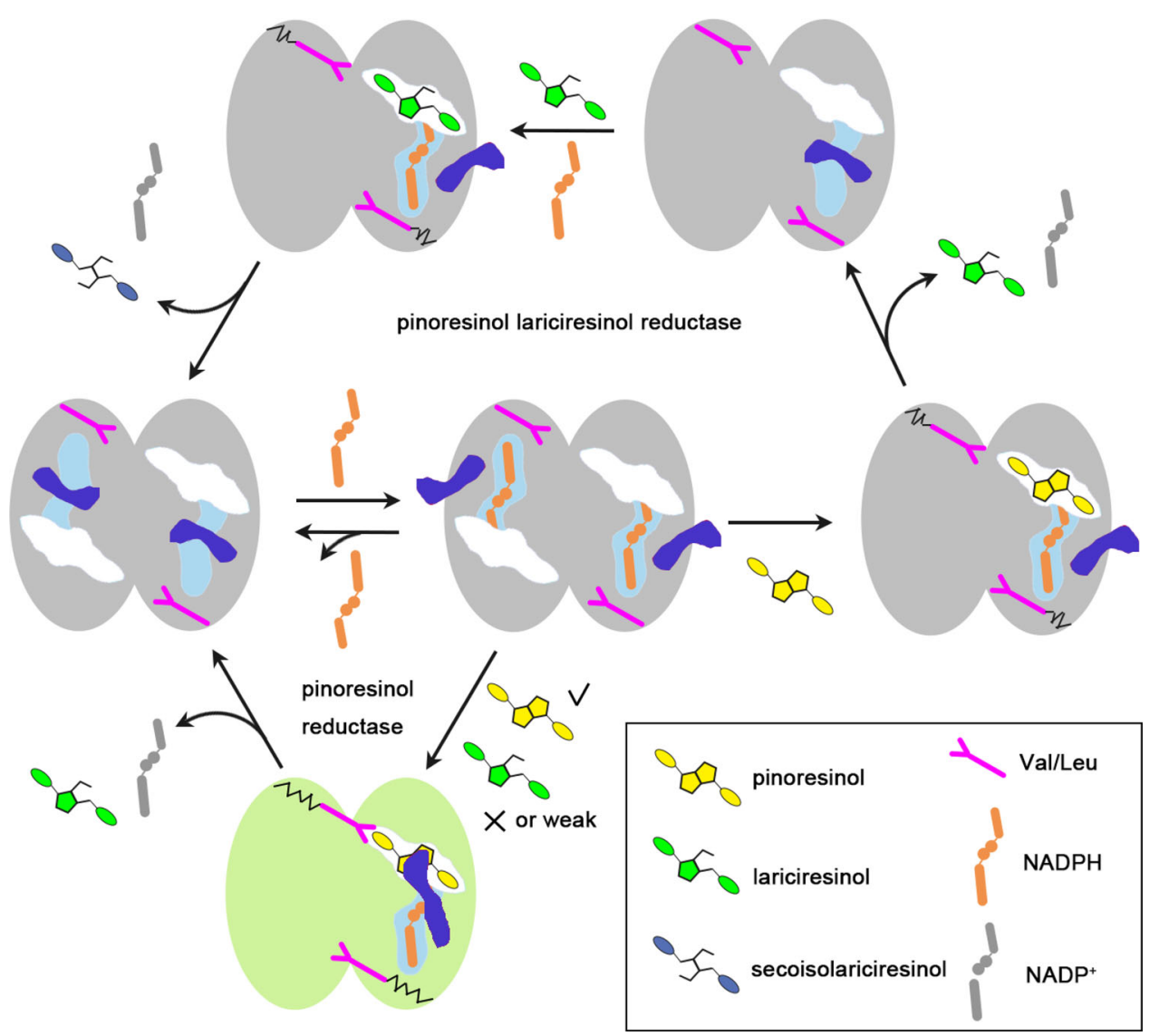

Fig. 5 Model depicting the catalytic processes of PLRs and PrRs. The blue dumbbell-shaped objects represent switches composed of $\beta 4$ loops. A movie showing how the enzymes change conformation throughout a single round of catalysis can be found in Supplementary Movie 1.

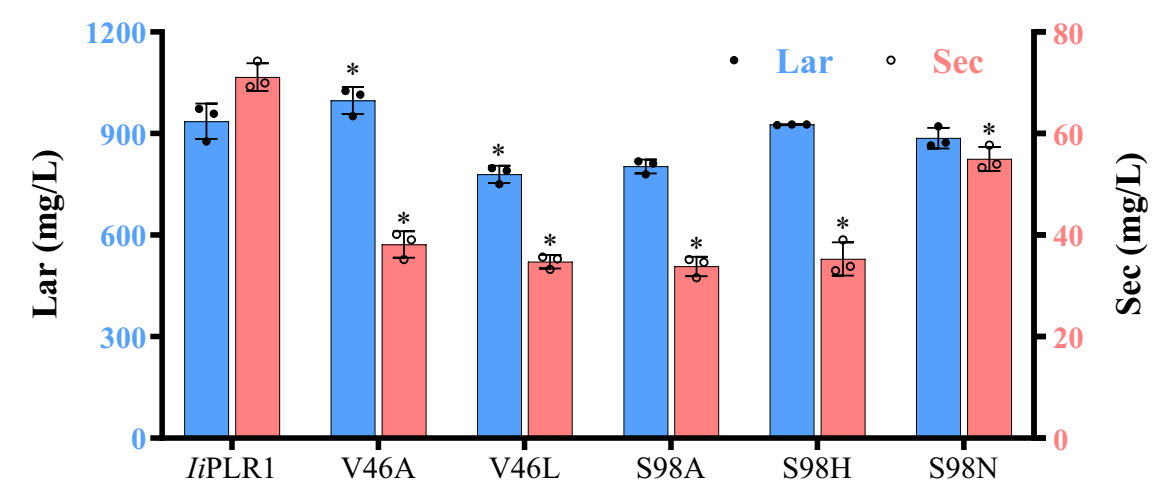

Fig. 6 Lariciresinol production through co-culture of different strains harboring a plasmid encoding liPLR1 or its single-site mutants with pinoresinolproducing cells. Data are mean \pm s.d. $\left(n=3\right.$ independent experiments). Asterisk $\left(^{*}\right)$ indicates significant difference from the wild-type enzyme $(P<0.05)$ analyzed by one-way ANOVA with Tukey's multiple comparisons test. Source data are provided as a Source Data file. 
complex with their various substrates. Several residues participating in substrate binding and catalysis were identified either directly or indirectly based on structural analysis, and these residues were validated by enzyme assays. All these data provide solid evidence to explore the mechanistic basis of substrate selectivity for PLRs/PrRs. Besides residues 46 and 98 in IiPLR1 and AtPrRs that we identified as being critical for binding and catalysis, residues Phe166, Tyr169, Phe170, His276, and Phe277 within the substrate-binding groove were also strongly correlated with enhanced substrate binding and catalysis. We further deduced that any residue in PLRs/PrRs around the hydrophobic groove or affecting homodimerization may impact the conformation of the active site, thereby dictating substrate selectivity (Supplementary Figs. 5 and 6). Consequently, it is not difficult to understand why mutant L174I of C. sinensis PLR1 can hardly reduce pinoresinol and specifically catalyze the conversion of lariciresinol to secoisolariciresinol ${ }^{16}$, i.e., because Leu174 points directly toward Tyr163 and thus may indirectly promote substrate recognition.

In addition, PLRs also display substrate stereochemical selectivity, which contributes to the enantiomeric diversity of lignans ${ }^{21-24}$. We found this to be true for IiPLR1, which gave comparable $k_{\text {cat }} / K_{\mathrm{m}}$ values for both $( \pm)$-pinoresinol and $( \pm)$-lariciresinol in the range of $0.9-1.6 \mu \mathrm{M}^{-1} \mathrm{~min}^{-1}$, although no experiments with respect to the enantio-specificity of this enzyme have been performed ${ }^{12,18}$. Despite past research on this topic, however, the mechanism underlying the substrate stereochemical selectivity of PLRs remains unclear. Comparison of the enantiospecifically opposite PLRs TpPLR1 and TpPLR2 suggests that F164, V268, and L272 in TpPLR1 contribute to the catalysis of (-)-pinoresinol, whereas L164, G268, and F272 in TpPLR2 prefer to bind $(+)$-pinoresinol ${ }^{17}$. Nevertheless, site-directed mutagenesis carried out in flax indicates that these positions are insufficient to determine enantiospecificity ${ }^{21}$. Based on amino-acid sequence and structural analyses, it seems that residues Phe94 and Phe277 in IiPLR1/AtPrR1 may act in concert to determine the enantiospecificity of PLRs (Supplementary Fig. 6). These two residues are highly conserved in PLRs that have no enantio-specificity, whereas Ile94 and Tyr277 are present in PLRs that have a strict substrate preference for $(+)$-pinoresinol. Moreover, in Linum usitatissimum PLR1 ( $L$ PLR1), which has strict enantiospecificity for $(-)$-pinoresinol, a leucine residue is deleted as are two other residues on $\beta 4$ loop (corresponding to the $\beta 4$ loop of IiPLR1, where Phe94 is located). Unfortunately, we could not obtain sufficient amounts of the enantiomerically pure substrates to carry out the experiments necessary to establish the enantioselectivity.

Nature uses a dazzling array of enzymes to produce diverse natural products. However, some modifications are challenging to control because the relative lack of substrate specificity often generates undesired byproducts. IiPLR1 plays an important role in the biotechnological production of lariciresinol ${ }^{18}$, which represents the most important component for the antibacterial, antiviral, and the immune-regulatory effects of the traditional Chinese medicine Radix Isatidis $3,25,26$. The fact that IiPLR1 can efficiently utilize both pinoresinol and lariciresinol as substrates ${ }^{18}$ suggests that the biosynthetic efficiency towards the pharmaceutically valuable compound lariciresinol in Radix Isatidis has been hampered by the relatively low substrate specificity of IiPLR1. In our present work, structure-guided mutagenesis successfully switched the substrate specificity of $I i$ PLR1, leading to overproduction of lariciresinol and reduced production of secoisolariciresinol by E. coli. Our study provides insight into the molecular mechanism underlying the substrate specificity of PLRs/PrRs, and paves the way for the manufacture of lariciresinol through microbial fermentation. Moreover, this work suggests the possibility of using targeted mutagenesis of IiPLR to improve the efficiency of synthesizing bioactive compounds in I. indigotica using gene-editing technologies ${ }^{27,28}$.

\section{Methods}

Phylogenic analysis of plant PLRs. Phylogenetic relationships were analyzed using the maximum likelihood method with the pairwise deletion option in MEGA 6.06. Tree reliability was estimated using a bootstrap analysis of 1000 replicates $^{29}$. Plant PLR amino-acid sequences used in the phylogenic analysis were retrieved from GenBank, including TpPLR1 (AAF63507.1), TpPLR2 (AAF63508.1), TpPLR3 (AAF63509.1), TpPLR4 (AAF63510.1), PpPLR (AHL21381.1), LaPLR1 (CAH60857.1), LuPLR1 (CAH60858.1), LuPLR2 (ABW24501.1), LpPLR1 (ABM68630.1), LcPLR1 (ABW86959.1), PhPLR (ACF71492.1), TcPLR1 (AZL88516.1), TcPLR2 (AZL88517.1), FiPLR1 (AAC49608.1), IiPLR1 (AEA42007.1), AtPrR1 (NP_174490.1), and AtPrR2 (NP_193102.1).

Heterologous expression of IiPLR1, AtPrR1, and AtPrR2 in E. coli. Total RNA was extracted from leaves of wild-type I. indigotica or A. thaliana using TRIzol Reagent (GIBCO BRL). The mRNA was reverse transcribed with oligo dT to generate cDNA as a template for PCR. Full-length cDNA sequences of IiPLR1 (GenBank accession no. JF264893), AtPrR1 (AY065214) and AtPrR2 (BT002882) were cloned into pET-duet-1 (Novagen, USA) to generate IiPLR1-pET, AtPrR1$\mathrm{pET}$, and AtPrR2-pET, respectively. The primers used are listed in Supplementary Table 1. E. coli Rosetta (DE3) cells were transformed with purified plasmid DNA and then grown at $37^{\circ} \mathrm{C}$ to an $\mathrm{OD}_{600}$ of 0.8 . Then, protein expression was induced by adding $0.5 \mathrm{mM}$ isopropyl $\beta$-D-thiogalactoside (IPTG, final concentration) with incubation overnight at $16^{\circ} \mathrm{C}$. Cells were collected, resuspended in buffer A (20 $\mathrm{mM}$ Tris- $\mathrm{HCl} \mathrm{pH} 8.0,100 \mathrm{mM} \mathrm{NaCl}$ ), and lysed with a French press. The lysate was centrifuged at $20,000 \times g$ for $45 \mathrm{~min}$, and the supernatant was applied to a $\mathrm{Ni}$ NTA column equilibrated with buffer A supplemented with $25 \mathrm{mM}$ imidazole. Bound protein was eluted using buffer A containing $250 \mathrm{mM}$ imidazole and was concentrated for further purification on a Superdex-200 column equilibrated with buffer A. Protein purity was assessed by SDS-PAGE (12\% polyacrylamide), and protein concentration was determined by the Bradford method ${ }^{30}$

Crystallization, data collection, and structure determination. The full-length IiPLR1/AtPrR1/AtPrR2 were purified as described above and concentrated to 5-10 $\mathrm{mg} \mathrm{mL}^{-1}$ for crystallization. Aliquots of each concentrated protein sample were mixed 1:1 with reservoir solution, and crystals were grown at 20 or $4{ }^{\circ} \mathrm{C}$ in one week using the sitting-drop vapor-diffusion method. For co-crystals, protein was combined with $\mathrm{NADP}^{+}$at a 1:5 molar ratio, and protein with $\mathrm{NADP}^{+}$and substrate/product at a 1:5:10 molar ratio. For reservoir solutions, IiPLR1 apo and cocrystals were grown with $0.2 \mathrm{M}$ sodium citrate tribasic, $0.1 \mathrm{M}$ sodium citrate/citric acid, pH 4.0 and 20\% polyethylene glycol (PEG) 3350; AtPrR1 apo crystals were grown in $0.2 \mathrm{M}$ lithium chloride, 20\% w/v PEG 3350; AtPrR1_NAP, AtPrR1 NAP_+ PIN, AtPrR1_NAP_+ LAR and AtPrR1_NAP_-SEC were grown in $0.2 \mathrm{M}$ sodium fluoride, $20 \%$ w/v PEG 3350; AtPrR1_NAP_-PIN and AtPrR1_NAP_LAR crystals were grown in $0.2 \mathrm{M}$ sodium malonate, $\mathrm{pH}$ 6.0, 20\% w/v PEG 3350; AtPrR2 apo crystals were grown in $0.2 \mathrm{M}$ magnesium chloride, $0.1 \mathrm{M}$ sodium HEPES, $\mathrm{pH}$ 7.5 and 25\% PEG 3350; AtPrR2_NAP_+ PIN crystals were grown in $2.1 \mathrm{M} \mathrm{DL}$ malic acid, $\mathrm{pH}$ 7.0. The crystals were cryoprotected by serial transfers into reservoir solutions supplemented with $30 \%(\mathrm{v} / \mathrm{v})$ glycerol and then flash-cooled in liquid nitrogen. Data collections were performed at the BL17U1 and BL19U1 beamline of the Shanghai Synchrotron Radiation Facility. The data were processed with HKL $3000^{31}$, and the initial phase was determined by molecular replacement with Phenix ${ }^{32}$ using the crystal structure of TpPLR1 (PDB ID: 1QYD [https://doi.org/ $10.2210 / \mathrm{pdb} 1 \mathrm{qyd} / \mathrm{pdb}]$ ) as a template. The structure models were firstly auto-built in $\mathrm{Coot}^{33}$ and then refined by iterative rounds of manual adjustment with Coot and refinement with Phenix. The statistics of data collection and structure refinement are shown in Supplementary Tables 2-5.

\section{Site-directed mutagenesis of liPLR1, AtPrR1, and AtPrR2 and enzymatic} assays. Single-site mutagenesis was achieved through one-step PCR, and mutants were verified with Sanger sequencing. All primers are listed in Supplementary Table 1. After expression and purification of recombinant enzymes under the aforementioned conditions, the results for the enzyme assays for mutants were compared with those for wild-type recombinant IiPLR1, AtPrR1, and AtPrR2 as follows.

Enzyme activity assays were conducted strictly according to our previous work ${ }^{18}$ Assay mixtures $(1 \mathrm{~mL})$ consisted of TG buffer $(50 \mathrm{mM}$ Tris- $\mathrm{HCl}, 10 \%$ [w/v] glycerol, $\mathrm{pH} 7.0), 150 \mu \mathrm{M}$ NADPH], $100 \mu \mathrm{M}$ pinoresinol, or $100 \mu \mathrm{M}$ lariciresinol and $5 \mu \mathrm{g}$ of purified protein. Assays without a fusion protein were used as controls. Protein, buffer, and substrate were pre-incubated for $5 \mathrm{~min}$ at $30^{\circ} \mathrm{C}$, and each reaction was initiated by addition of NADPH and terminated after 30 min by addition of $300 \mu \mathrm{L}$ ethyl acetate. Each assay mixture was extracted with ethyl acetate $(3 \times 300 \mu \mathrm{L}$ total $)$ The combined ethyl acetate phases were dried under vacuum, and the residue was dissolved in $1 \mathrm{~mL}$ methanol. Conversion rate was then determined. The content of pinoresinol, lariciresinol and secoisolariciresinol was determined by LC-MS using a triple-quadrupole mass spectrometer (Model 6410, Agilent, Santa Clara, CA) 
following our published methods ${ }^{18}$. MassHunter Qualitative Analysis B.06.00 was used for the data analysis. The selected transitions of $\mathrm{m} / \mathrm{z}$ were $357 \rightarrow 151$ for pinoresinol, $359 \rightarrow 329$ for lariciresinol, and $361 \rightarrow 164$ for secoisolariciresinol. All standards were purchased from Sigma-Aldrich (St. Louis, MO).

For determination of $V_{\max }$ and $K_{\mathrm{m}}$ values for $I i \mathrm{PLR} 1 \_\mathrm{V} 46 \mathrm{~A}, 10$ different concentrations of substrate (pinoresinol or lariciresinol; $5-200 \mu \mathrm{M}$ ) and $1 \mu \mathrm{g}$ purified protein were used. Samples were incubated at $30^{\circ} \mathrm{C}$ for $5 \mathrm{~min}$ (during which substrate consumption was $\leq 10 \%$ ). Samples without protein were used as controls. The rate of substrate consumption was calculated for kinetic analysis. $V_{\max }$ and $K_{\mathrm{m}}$ values were determined from Lineweaver-Burk plots, and $k_{\text {cat }}$ was determined by dividing $V_{\max }$ by the enzyme concentration.

Bioconversion. For the production of lariciresinol, biotransformation was divided into two modules, namely the accumulation and conversion of the precursor, pinoresinol. E. coli strain strOpr2 carrying plasmid pET28a-Prx02-PsVAO was used to produce pinoresinol ${ }^{19}$, whereas E. coli BL21(DE3) carrying a plasmid encoding IiPLR1 or its mutants was used for conversion of pinoresinol to lariciresinol. These E. coli strains were cultured in $\mathrm{LB}$ medium at $37^{\circ} \mathrm{C}$ with shaking $(220 \mathrm{rpm})$ for $12 \mathrm{~h}$ as seed cultures, and then a $2 \%$ seed culture was transferred to a $250-\mathrm{mL}$ shaker flask containing $25 \mathrm{~mL}$ TB medium. After culturing for $2-2.5 \mathrm{~h}$ at $37^{\circ} \mathrm{C}$ and $220 \mathrm{rpm}, 500$ $\mu \mathrm{M}$ IPTG (final concentration) was added to the medium with continued cultivation for $12 \mathrm{~h}$ at $25^{\circ} \mathrm{C}$ and $220 \mathrm{rpm}$. These cells were used for pinoresionol accumulation and conversion, respectively. Cells from E. coli strain strOpr2 were harvested by centrifugation at $4{ }^{\circ} \mathrm{C}$ and $3724 \times g$ for $30 \mathrm{~min}$ and then resuspended in phosphatebuffered saline $(\mathrm{pH} 7.0)$ to adjust the $\mathrm{OD}_{600}$ value to 20 . Then $0.15 \%(\mathrm{v} / \mathrm{v})$ eugenol was added into $15 \mathrm{~mL}$ of the resuspension at $0,1,3,5$, and $7 \mathrm{~h}$ for pinoresinol accumulation $\left(20^{\circ} \mathrm{C}, 220 \mathrm{rpm}\right)$. At $9 \mathrm{~h}, 15 \mathrm{ml}$ of a culture of $E$. coli expressing $I i P L R 1$ and each mutant $\left(\mathrm{OD}_{600}=20\right)$ was added to determine the conversion of pinoresinol to lariciresinol $\left(25^{\circ} \mathrm{C}, 220 \mathrm{rpm}\right)$, and samples were taken after $20 \mathrm{~h}$. The concentration of each of lariciresinol and secoisolariciresinol was determined by HPLC.

Statistical analysis. All the experiments in this paper were repeated at least three times and results from representative data sets are presented. GraphPad Prism (version 9.1.0) was used for the statistical analysis. The statistical evaluations used one-way analysis of variance (ANOVA) with multiple comparisons, followed by Tukey tests. The results were considered statistically significant at ${ }^{*} P<0.05$.

Reporting summary. Further information on research design is available in the Nature Research Reporting Summary linked to this article.

\section{Data availability}

Data supporting the findings of this work are available within the paper and its Supplementary Information files. The atomic coordinates and structure factors for the structures have been deposited in the Protein Data Bank with accession codes 7CS2 (Apo structure of dimeric IiPLR1), 7CS3 (IiPLR1 with NADP ${ }^{+}$), 7CS4 (IiPLR1 with NADP ${ }^{+}$ and (+)pinoresinol), 7CS5 (IiPLR1 with $\mathrm{NADP}^{+}$and (-)pinoresinol), 7CS6 (IiPLR1 with $\mathrm{NADP}^{+}$and $(-)$lariciresinol), $7 \mathrm{CS} 7\left(\right.$ IiPLR1 with $\mathrm{NADP}^{+}$and $(+)$ secoisolariciresinol), 7CS8 (IiPLR1 with $\mathrm{NADP}^{+}$and (-)secoisolariciresinol), 7CS9 (AtPrR1 in apo form), 7CSA (AtPrR1 with NADP $\left.{ }^{+}\right), 7 \mathrm{CSB}\left(\right.$ AtPrR1 with $\mathrm{NADP}^{+}$and (+)pinoresinol), 7CSC (AtPrR1 with $\mathrm{NADP}^{+}$and (-)pinoresinol), 7CSD (AtPrR1 with $\mathrm{NADP}^{+}$and $(+)$lariciresinol), 7CSE $\left(\right.$AtPrR1 with $\mathrm{NADP}^{+}$and $(-)$lariciresinol), $7 \mathrm{CSF}$ (AtPrR1 with $\mathrm{NADP}^{+}$and $(-)$secoisolariciresinol), 7CSG (AtPrR2 in apo form), 7CSH $\left(A t \operatorname{PrR} 2\right.$ with $\mathrm{NADP}^{+}$and $(+)$pinoresinol). The initial phase was determined by molecular replacement using the crystal structure of TpPLR1 (PDB ID: 1QYD [https:// doi.org/10.2210/pdblqyd/pdb]) as a template. The source data underlying Figs. $2 \mathrm{~g}, 4 \mathrm{a}, \mathrm{b}$, and 6, as well as Table 2 are provided as a Source Data file. All data generated and analyzed during the current study are available from the corresponding authors upon reasonable request. A reporting summary for this Article is available as a Supplementary Information file. Source data are provided with this paper.

Received: 10 July 2020; Accepted: 14 April 2021;

Published online: 14 May 2021

\section{References}

1. Teponno, R. B., Kusari, S. \& Spiteller, M. Recent advances in research on lignans and neolignans. Nat. Prod. Rep. 33, 1044-1092 (2016).

2. Teodor, E. D., Moroeanu, V. \& Radu, G. L. Lignans from medicinal plants and their anticancer effect. Mini Rev. Med. Chem. 20, 1083-1090 (2020).

3. J, L. et al. Lariciresinol-4-O- $\beta$-D-glucopyranoside from the root of Isatis indigotica inhibits influenza A virus-induced pro-inflammatory response. J. Ethnopharmacol. 174, 379-386 (2015).

4. Xiao, P., Huang, H., Chen, J. \& Li, X. In vitro antioxidant and antiinflammatory activities of Radix Isatidis extract and bioaccessibility of six bioactive compounds after simulated gastro-intestinal digestion. $J$. Ethnopharmacol. 157, 55-61 (2014).

5. Hirano, T., Wakasugi, A., Oohara, M., Oka, K. \& Sashida, Y. Suppression of mitogen-induced proliferation of human peripheral blood lymphocytes by plant lignans. Planta Med. 57, 331-334 (1991).

6. Haihan et al. Two new furofuran lignans from Kandelia obovata. Heterocycles 87, 1093-1098 (2013).

7. Chin, Y., Chai, H., Keller, W. J. \& Kinghorn, A. D. Lignans and other constituents of the fruits of Euterpe oleracea (Acai) with antioxidant and cytoprotective activities. J. Agric. Food Chem. 56, 7759-7764 (2008).

8. Jang, Y. P., Kim, S. R. \& Kim, Y. C. Neuroprotective dibenzylbutyrolactone lignans of Torreya nucifera. Planta Med. 67, 470-472 (2001).

9. Canel, C., Moraes, R. M., Dayan, F. E. \& Ferreira, D. Podophyllotoxin. Phytochemistry 54, 115-120 (2000).

10. Lau, W. \& Sattely, E. S. Six enzymes from mayapple that complete the biosynthetic pathway to the etoposide aglycone. Science 349, 1224-1228 (2015).

11. Laurence et al. Stereoselective bimolecular phenoxy radical coupling by an auxiliary (dirigent) protein without an active center. Science 275, 362-366 (1997)

12. Markulin, L. et al. Pinoresinol-lariciresinol reductases, key to the lignan synthesis in plants. Planta 249, 1695-1714 (2019).

13. Dinkova-Kostova, A. T. et al. (+)-Pinoresinol/(+)-lariciresinol reductase from Forsythia intermedia. Protein purification, cDNA cloning, heterologous expression and comparison to isoflavone reductase. J. Biol. Chem. 271, 29473-29482 (1996).

14. Umezawa, T. Diversity in lignan biosynthesis. Phytochem. Rev. 2, 371-390 (2003).

15. Tomoyuki, N., Masaharu, M., Shiro, S., Takefumi, H. \& Toshiaki, U. Characterization of Arabidopsis thaliana pinoresinol reductase, a new type of enzyme involved in lignan biosynthesis. J. Biol. Chem. 283, 15550-15557 (2008).

16. $\mathrm{Wu}, \mathrm{Y}$. et al. A variable loop involved in the substrate selectivity of pinoresinol/ lariciresinol reductase from Camellia sinensis. Phytochemistry 162, 1-9 (2019).

17. Tongpil, M. et al. Crystal structures of pinoresinol-lariciresinol and phenylcoumaran benzylic ether reductases and their relationship to isoflavone reductases. J. Biol. Chem. 278, 50714-50723 (2003).

18. Xiao, Y. et al. Combined transcriptome and metabolite profiling reveals that IiPLR1 plays an important role in lariciresinol accumulation in Isatis indigotica. J. Exp. Bot. 66, 6259-6271 (2015).

19. Lv, Y., Cheng, X., Du, G., Zhou, J. \& Chen, J. Engineering of an $\mathrm{H}_{2} \mathrm{O}_{2}$ autoscavenging in vivo cascade for pinoresinol production. Biotechnol. Bioeng. 114, 2066-2074 (2017)

20. Decembrino, D., Girhard, M. \& Urlacher, V. B. Use of copper as a trigger for in vivo activity of $E$. coli laccase CueO: a simple tool for biosynthetic purposes. ChemBioChem. 22, 1470-1479 (2021).

21. Heimendahl, C. B. I. V. et al. Pinoresinol-lariciresinol reductases with different stereospecificity from Linum album and Linum usitatissimum. Phytochemistry 66, 1254-1263 (2005).

22. Chu, A., Dinkova, A., Davin, L. B., Bedgar, D. L. \& Lewis, N. G. Stereospecificity of $(+)$-pinoresinol and $(+)$-lariciresinol reductases from Forsythia intermedia. J. Biol. Chem. 268, 27026-27033 (1993).

23. Fujita, M., Gang, D. R., Davin, L. B. \& Lewis, N. G. Recombinant pinoresinollariciresinol reductases from western red cedar (Thuja plicata) catalyze opposite enantiospecific conversions. J. Biol. Chem. 274, 618-627 (1999).

24. Sicilia, T., Niemeyer, H. B., Honig, D. M. \& Metzler, M. Identification and stereochemical characterization of lignans in flaxseed and pumpkin seeds. J. Agric. Food Chem. 51, 1181-1188 (2003).

25. Yang, Z. et al. Antiviral activity of Isatis indigotica root-derived clemastanin B against human and avian influenza $\mathrm{A}$ and $\mathrm{B}$ viruses in vitro. Int. J. Mol. Med. 31, 867-873 (2013).

26. Council, N. P. Pharmacopoeia of China 2015 Edition. (Chemical Industry, 2015)

27. Anzalone, A. V. et al. Search-and-replace genome editing without doublestrand breaks or donor DNA. Nature 576, 149-157 (2019).

28. Lin, Q. et al. Prime genome editing in rice and wheat. Nat. Biotechnol. 38, 582-585 (2020).

29. Tamura, K. et al. MEGA5: molecular evolutionary genetics analysis using maximum likelihood, evolutionary distance, and maximum parsimony methods. Mol. Biol. Evol. 28, 2731-2739 (2011).

30. Bradford, M. M. A rapid and sensitive method for the quantitation of microgram quantities of protein utilizing the principle of protein-dye binding. Anal. Biochem. 72, 248-254 (1976).

31. Cymborowski, M., Otwinowski, Z., Chruszcz, M. \& Minor, W. HKL-3000: the integration of data reduction and structure solution-from diffraction images to an initial model in minutes. Acta Crystallogr. D D62, 859-866 (2006).

32. Adams, P. D. et al. PHENIX: a comprehensive Python-based system for macromolecular structure solution. Acta Crystallogr. D D66, 213-221 (2010).

33. Emsley, P. \& Cowtan, K. Coot: model-building tools for molecular graphics. Acta Crystallogr. D D60, 2126-2132 (2004). 


\section{Acknowledgements}

This work was sponsored by National Key R\&D Program of China (2019YFC1711100, 2018YFA0900600, 2019YFC1711000, SQ2018YFA090071), National Natural Science Foundation of China $(81874335,32025020,31872665,31670292$ and 31970316), the National Science Fund for Excellent Young Scholars (21822806), Shanghai Rising-Star Program (18QB1402700), the Chinese Academy of Sciences Strategic Priority Research Program (XDB27020103), Shanghai local Science and Technology Development Fund Program guided by the Central Government (YDZX20203100002948), and Shanghai Science and technology Commission (19XD1424500). We thank the staff members at SSRF BL17U1 and BL19U1 for their technical assistance in X-ray diffraction data collection, and the core facility center of Institute of Plant Physiology and Ecology (CAS) for $\mathrm{X}$-ray diffraction test.

\section{Author contributions}

Y.X., W.C., K.S., P.Z., J.Z., and L.Z. designed experiments. K.S. carried out protein expression, purification, crystallization, structure determination, and data analysis. Y.X., X.M., Y.Y., J.C., J.F., S.Q., and Z.L. carried out enzymatic assays. J.Z., L.W., and D. W. performed bioconversion and optimization. Y.X., K.S., J.Z., W.C., P.Z., and L.Z. analyzed the data and wrote the manuscript.

\section{Competing interests}

The authors declare no competing interests.

\section{Additional information}

Supplementary information The online version contains supplementary material available at https://doi.org/10.1038/s41467-021-23095-y.
Correspondence and requests for materials should be addressed to L.Z., P.Z. or W.C.

Peer review information Nature Communications thanks Clint Chapple, Jiahai Zhou, and other, anonymous, reviewers for their contributions to the peer review of this work. Peer review reports are available.

Reprints and permission information is available at http://www.nature.com/reprints

Publisher's note Springer Nature remains neutral with regard to jurisdictional claims in published maps and institutional affiliations.

cC (i) Open Access This article is licensed under a Creative Commons Attribution 4.0 International License, which permits use, sharing, adaptation, distribution and reproduction in any medium or format, as long as you give appropriate credit to the original author(s) and the source, provide a link to the Creative Commons license, and indicate if changes were made. The images or other third party material in this article are included in the article's Creative Commons license, unless indicated otherwise in a credit line to the material. If material is not included in the article's Creative Commons license and your intended use is not permitted by statutory regulation or exceeds the permitted use, you will need to obtain permission directly from the copyright holder. To view a copy of this license, visit http://creativecommons.org/ licenses/by/4.0/.

(C) The Author(s) 2021 